KFA-IKP(Th)-1994-6

24 January 1994

\title{
Deep inelastic scattering at HERA and the BFKL pomeron
}

\author{
N.N. Nikolaev ${ }^{a, b}$, and B.G. Zakharov ${ }^{b}$ \\ ${ }^{a}$ IKP(Theorie), KFA Jülich, 5170 Jülich, Germany \\ ${ }^{b}$ L. D. Landau Institute for Theoretical Physics, GSP-1, 117940, \\ ul. Kosygina 2, Moscow 117334, Russia.
}

\begin{abstract}
We present a BFKL phenomenology of structure functions in the framework of the generalized BFKL equation for the dipole cross section proposed by us recently. We show that the HERA measurements of excitation of charm at $Q^{2} \lesssim 10 \mathrm{GeV}^{2}$ allow determination of the intercept of the BFKL pomeron. A comparison with the experimental data suggests a large contribution from the nonperturbative cross section for colour dipoles of large size $r$.
\end{abstract}

E-mail: kph154@zam001.zam.kfa-juelich.de 
The $S$-matrix of diffractive scattering is exactly diagonalized in terms of the dipole cross section [1-3] which satisfies the generalized BFKL (Balitskii-Fadin-Kuraev-Lipatov) equation derived in [4-7]. In [6,7] we have determined the intercept of the pomeron $\Delta_{\mathbf{I P}}$ and found the pomeron dipole cross section in the realistic model with the running QCD coupling $\alpha_{S}(r)$ and the finite correlation radius $R_{c}$ for perturbative gluons. With the $\mu_{G}=1 / R_{c}=0.75 \mathrm{GeV}$ and the infrared freezing of the strong coupling $\alpha_{S}\left(r \geq R_{f}\right)=\alpha_{S}^{(f r)}=0.8$ we found $\Delta_{\mathbb{P}}=0.4$ $[6]$.

In this paper we discuss the coresponding BFKL phenomenology of deep inelastic scattering at large $\frac{1}{x}$, where $x$ is the Bjorken variable. The starting point of our analysis is the dipole-cross section representation for the virtual photoabsorption cross section [1]

$$
\sigma_{T, L}\left(\gamma^{*} N, \xi, Q^{2}\right)=\int_{0}^{1} d z \int d^{2} \vec{r}\left|\Psi_{T, L}(z, r)\right|^{2} \sigma(\xi, r)
$$

where $\sigma(\xi, r)$ is the dipole cross section for the colour dipole of size $r, Q^{2}$ is the virtuality of the photon, $\xi=\log \left(\frac{x_{0}}{x}\right)$ and $x_{0} \sim 0.1-0.01$ corresponds to the onset of the leading- $\log \left(\frac{1}{x}\right)$ approximation. In the perturbative part of the dipole cross section $\sigma^{(p t)}(\xi, r)$, which is a solution of our generalized BFKL equation, the gluon correlation radius $R_{c}$ emerges as the principal parameter which controls its $r$ dependence and the absolute normalization. At $r>R_{c}$ the dipole cross section contains also the nonperturbative component, which is of major concern from the point of view of the phenomenology to be presented in this paper. The wave functions of the $(\mathrm{T})$ transverse and $(\mathrm{L})$ longitudinal virtual photon were derived in [1] and read

$$
\begin{gathered}
\left|\Psi_{T}(z, r)\right|^{2}=\frac{6 \alpha_{e m}}{(2 \pi)^{2}} \sum_{1}^{N_{f}} e_{f}^{2}\left\{\left[z^{2}+(1-z)^{2}\right] \varepsilon^{2} K_{1}(\varepsilon r)^{2}+m_{f}^{2} K_{0}(\varepsilon r)^{2}\right\} \\
\left|\Psi_{L}(z, r)\right|^{2}=\frac{6 \alpha_{e m}}{(2 \pi)^{2}} \sum_{1}^{N_{f}} 4 e_{f}^{2} Q^{2} z^{2}(1-z)^{2} K_{0}(\varepsilon r)^{2}
\end{gathered}
$$

where $K_{1}(x)$ is the modified Bessel function, $e_{f}$ is the quark charge in units of the electron charge, $\varepsilon^{2}=z(1-z) Q^{2}+m_{f}^{2}, m_{f}$ is the quark mass and $z$ is the fraction of photon's (lightcone) momentum carried by the quark of the $q \bar{q}$ pair $(0<z<1)$. Then, the sea structure function is calculated as $F_{2}\left(x, Q^{2}\right)=Q^{2}\left[\sigma_{T}+\sigma_{L}\right] /\left(4 \pi^{2} \alpha_{e m}\right)$. The dipole cross section $\sigma(\xi, r)$ is universal, the $Q^{2}$ and the flavour dependence of structure functions only comes from $\left|\Psi_{T, L}\right|^{2}$. 
The derivation [1] of (11) uses the observation that at $\frac{1}{x} \gg 1$, the virtual photoabsorption can be viewed as interaction with the target proton of the multipartonic lightcone Fock states $(q \bar{q}, q \bar{q} g \ldots)$ of the photon, which are formed at large distance $\Delta z \sim \frac{1}{m_{p} x}$ upstream the target. In [4] we gave the regular procedure for construction of the corresponding multiparton lightcone wave function and of the multiparton cross section. The Fock states with $n$ soft gluons give the $\propto \xi^{n}$ contribution to the total photoabsorption cross section, which can be reabsorbed into the energy dependent perturbative dipole cross section

$$
\sigma^{(p t)}(\xi, r)=\sum_{n=0} \frac{1}{n !} \sigma_{n}(r) \xi^{n},
$$

which satisfies the generalized BFKL equation [2-5]

$$
\frac{\partial \sigma^{(p t)}(\xi, r)}{\partial \xi}=\mathcal{K} \otimes \sigma^{(p t)}(\xi, r)
$$

where in terms of the expansion (4) the kernel $\mathcal{K}$ is defined by

$$
\begin{gathered}
\sigma_{n+1}(r)=\mathcal{K} \otimes \sigma_{n}(r)= \\
\frac{3}{8 \pi^{3}} \int d^{2} \vec{\rho}_{1} \mu_{G}^{2}\left|g_{S}\left(R_{1}\right) K_{1}\left(\mu_{G} \rho_{1}\right) \frac{\vec{\rho}_{1}}{\rho_{1}}-g_{S}\left(R_{2}\right) K_{1}\left(\mu_{G} \rho_{2}\right) \frac{\vec{\rho}_{2}}{\rho_{2}}\right|^{2}\left[\sigma_{n}\left(\rho_{1}\right)+\sigma_{n}\left(\rho_{2}\right)-\sigma_{n}(r)\right] .
\end{gathered}
$$

Here $R_{c}=1 / \mu_{G}$ is the correlation radius for perturbative gluons, $\vec{\rho}_{2}=\vec{\rho}_{1}-\vec{r}, R_{i}=$ $\min \left\{r, \rho_{i}\right\}, g_{S}(r)$ is the effective colour charge,

$$
\alpha_{S}(r)=\frac{g_{S}(r)^{2}}{4 \pi}=\frac{4 \pi}{\beta_{0} \log \left(\frac{C^{2}}{\Lambda_{Q C D}^{2} r^{2}}\right)},
$$

where $\beta_{0}=11-\frac{2}{3} N_{f}=9$ for $N_{f}=3$ active flavours, $C \approx 1.5$ [1] and we impose the infrared freezing $\alpha_{S}\left(r>R_{f}\right)=\alpha_{S}^{(f r)}=0.8$ [6]. The correspondance to the original BFKL equation [8] was discussed in detail in [4-7].

Once the dipole cross section (4) is known, Eq. (1) enables the parameter-free calculation of the proton structure function at small $x$, which is the subject of the present communication. Below we discuss the salient features of the emerging BFKL phenomenology of deep inelastic scattering at small $x$. We start with the discussion of the nonperturbative component of the dipole cross section, which dominates at large $r$ and at moderately large $\frac{1}{x}$. Then, we present calculations of the proton structure function in the kinematical range 
of HERA and find good agreement with the data from ZEUS and H1 experiments $[9,10]$. We comment on determination of the pomeron intercept from the charm structure function. We also give an estimate of the gluon distributions which correspond to these structure functions and give predictions for $R=\sigma_{L} / \sigma_{T}$.

We start presentation of our results with the description of the colour dipole cross secton. In the perturbative QCD, the growth of the dipole cross section comes from the logarithmic growth of the multiplicity $n_{g}$ of perturbative guons in the photon generated from the parent $q \bar{q}$ Fock state $n_{g} \propto \int_{x}^{x_{0}} d z_{g} / z_{g}=\xi$. Equations $(5,6)$ describe the perturbative part of the dipole cross section: the kernel $\mathcal{K}$ is directly proportional to the probability of radiation of the perturbative gluons [4-7], and $\Delta \sigma_{g}=\frac{9}{8}\left[\sigma\left(\rho_{1}\right)+\sigma\left(\rho_{2}\right)-\sigma(r)\right]$ shows how much the interaction cross section is changed for the presense of the perturbative gluon (here $\vec{\rho}_{1,2}$ is the separation of the gluon from the (anti)quark, $\vec{\rho}_{2}=\vec{\rho}_{1}-\vec{r}$ and $\sigma(r)$ is the dipole cross section for the parent $q \bar{q}$-dipole of size $\vec{r}$ ). To higher orders, the gluons themselves become the source of the subsequent generation of gluons, so that Fock states with $n$ soft gluons give $\propto \xi^{n}$ contribution to the dipole cross section. As a boundary conditon at $x_{0}=3 \cdot 10^{-2}$, one can start with the two-gluon exchange dipole cross section for interaction with the nucleon target $[1]$

$$
\sigma_{0}(r)=\frac{32}{9} \int \frac{d^{2} \vec{k}}{\left(\vec{k}^{2}+\mu_{G}^{2}\right)^{2}} \alpha_{S}\left(k^{2}\right) \alpha_{S}\left(\kappa^{2}\right)\left[1-G_{p}\left(3 \vec{k}^{2}\right)\right][1-\exp (i \vec{k} \vec{r})],
$$

where $G_{p}\left(q^{2}\right)$ is the charge form factor of the proton, $\kappa^{2}=\max \left\{k^{2}, \frac{C^{2}}{r^{2}}\right\}$.

The rightmost singularity in the complex $j$-plane corresponds to the pomeron cross section $\sigma_{\mathbf{I P}}(\xi, r)=\sigma_{\mathbf{I P}}(r) \exp \left(\Delta_{\mathbf{I P}} \xi\right)$. The crucial point of the BFKL phenomenology is that the pomeron cross section $\sigma_{\mathbf{I P}}(r)$ is not, and can not, be confined to the small- $r$ region, which is due to the so-called diffusion property of the Green's function of the BFKL equation [8]. The underlying physics is very simple: even when one starts with the small-size beam and target colour dipoles, radiated gluons stick out of the parent dipoles the transverse distance $\sim R_{c}$ apart, and it is interaction of these gluons which controlls the total cross section. For instance, the pomeron intercept $\Delta_{\mathbf{I P}}$ is predominatly controlled by the behavior of $\sigma_{\mathbf{I P}}(r)$ in the semi-perturbative region of $r \sim R_{c}$ [6]. Consequently, the contribution from the infrared region of large $r$, and the infrared regularization of the perturbative QCD, are at the heart 
of the BFKL phenomenology.

Motivated by the lattice studies of the correlation function of perturbative gluons [11], we take $\mu_{G}=0.75 \mathrm{GeV}$. With such a large value of $\mu_{G}$, the driving term (8) of the perturbative dipole cross section $\sigma^{(p t)}(\xi=0, r)=\sigma_{0}(r)$ flattens at large $r$ at $\sim 8 \mathrm{mb}$ (Fig. 1$)$. One can easily anticipate the nonperturbative mechanism of interaction of large, $r \gtrsim R_{c}$, colour dipoles. Here we make an assumption that the perturbative and nonperturbative cross sections are additive [12]

$$
\sigma(\xi, r)=\sigma^{(p t)}(\xi, r)+\sigma^{(n p t)}(r) .
$$

Because the rise of the $\sigma^{(p t)}(\xi, r)$ is due to the rising multiplicity of the perturbative gluons in the photon wave function, we make a plausible assumption that the nonperturbative dipole cross section does not rise with energy. We also assume the dominance of the perturbative cross section, $\sigma^{(n p t)} / \sigma^{(p t)}(r) \ll 1$ at $r \ll R_{c}$. In Fig. 1 we show our choice of $\sigma^{(n p t)}(r)$.

Different processes probe $\sigma(\xi, r)$ at different $r$, which allows to disentangle the perturbative and nonperturbative dipole cross sections experimentally. For instance, we shall see that the charm production predominantly probes $\sigma^{(p t)}(\xi, r)$. The above choice of $\sigma^{(n p t)}(r)$ is already strongly constrained by various experimental data. As a matter of fact, we require that the sum of the perturbative and nonperturbative cross sections $\sigma(\xi=0, r)$ roughly reproduces the colour dipole cross section of Ref. 1, which was constrained to reproduce $\sigma_{\text {tot }}(\pi N) \approx 25 \mathrm{mb}$. The cross section of Ref. 1 has already been succesfully used in the calculation of the structure functions at moderate $Q^{2}$ (see also below) and of the nuclear shadowing $[1,3]$, of the total photoproduction cross section and of the rate of the diffraction dissociation of real and virtual photons [2]. The E665 data on the exclusive leptoproduction of the $\rho^{0}$-mesons on nuclei [13] and the NMC data on the $J / \Psi$ photoproduction on nuclei [14] imply that $\sigma(\xi=0, r)$ decreases from $\sim 30 \mathrm{mb}$ at $r \sim(1-1.5)$ f to $\sim 6 \mathrm{mb}$ at $r \sim 0.4 \mathrm{f}$ (for the detailed discussion see $[15,16])$.

Because the ratio $\sigma(\xi, r) / r^{2}$ only slowly increases towards small $r$, it is useful to rewrite Eq. (11) in the form

$$
F_{2}\left(x, Q^{2}=\frac{1}{\pi^{3}} \int \frac{d r^{2}}{r^{2}} \cdot \frac{\sigma(\xi, r)}{r^{2}} \Phi_{T}\left(Q^{2}, r^{2}\right) .\right.
$$

The divided by $e_{f}^{2}$ contributions of light and charmed quarks to the kernel $\Phi_{T}\left(Q^{2}, r^{2}\right)=$ 
$\left(\pi^{2} / 4 \alpha_{e m}\right) \int_{0}^{1} d z Q^{2} r^{4}\left|\Psi_{T, L}(z, r)\right|^{2}$ are shown in Fig. 2. They develope a plateau at large $Q^{2}$, the emergence of which signals the onset of the GLDAP (Gribov-Lipatov-DokshitzerAltarelli-Parisi) [17] evolution of parton densities. The Fig. 2 in conjunction with Fig. 1 shows which region of $r$ is probed in deep inelastic scattering at the virtuality $Q^{2}$. Following [1-3], we take the quark masses $m_{u, d}=150 \mathrm{MeV}, m_{s}=300 \mathrm{MeV}, m_{c}=1.5 \mathrm{GeV}, m_{b}=$ 4.5GeV. The structure functions are insensitive to $\sigma(\xi, r)$ at $r \gtrsim 2 \mathrm{f}$. Notice, that the contribution from $r \sim 1 \mathrm{f}$ is the perfectly scaling part of $F_{2}\left(x, Q^{2}\right)$ which persists at all the values of $Q^{2}$ [1]. Numerically, we find that the contribution from $\sigma^{(n p t)}(r)$ to $F_{2}\left(x, Q^{2}\right)$ changes form $F_{2}^{(n p t)}\left(x, Q^{2}=0.75 \mathrm{GeV}^{2}\right) \approx 0.1$ to $F_{2}^{(n p t)}\left(x, Q^{2}=15 \mathrm{GeV}^{2}\right) \approx 0.13$. In Fig. 3a we show the total sea structure function, in Fig. 3b we show its perturbative part. Because of expansion of the plateau in Fig. 2 with rising $Q^{2}$, the contribution to $F_{2}\left(x, Q^{2}\right)$ from $\sigma^{(p t)}(\xi, r)$ takes over at large $Q^{2}$. The values of structure function already at $Q^{2} \gtrsim(2$ 4) $\mathrm{GeV}^{2}$ are insensitive to masses of light quarks. Contribution from $\sigma^{(p t)}(\xi, r)$ also takes over at large $\frac{1}{x}$, but at $x \sim 10^{-2}$ the effect of $F^{(n p t)}\left(x, Q^{2}\right)$ is quite substantial.

In Fig. 4 we compare our predictions with the NMC [18], ZEUS [10] and H1 [11] structure functions. The overall agreement is good; the slight departure from the NMC data points at large $x$ is due to the the valence structure function not considered here. Besides, at large $x$ the nonperturbative cross section can contain the Regge-decreasing component $\propto$ $\exp \left(-\frac{1}{2} \xi\right)=\sqrt{x / x_{0}}$, for the magnitude of which we do not have any educated guess [19]. It is somewhat more accurate to use in Eq. (1) $\sigma(\xi+\log z, r)$, but for the purposes of the qualitative phenomenology of the present paper, this rescaling of the energy variable can be reabsorbed into renormalization of the boundary condition.

In [7] we have shown that the energy dependence of $\sigma^{(p t)}(\xi, r)$ at $r \sim 0.5 R_{c}$ follows the asymptotic law $\propto \exp \left(\Delta_{\mathbf{I P}} \xi\right)$ starting already at moderate energy. At $r \ll R_{c}$ the effective intercept is substantially larger than $\Delta_{\mathbb{P}}$ beacuse of the double-leading-logarithm effects [5,7]. Eq. (15) and Fig. 2 show that the total structure function $F_{2}\left(x, Q^{2}\right)$ receives contributions from a broad range of $r$. Besides, Figs. 3a and $3 \mathrm{~b}$ show that the $x$-dependence of $F_{2}\left(x, Q^{2}\right)$ is strongly affected by the contribution from the nonperturbative cross section, which does not allow the model-independent determination of the pomeron intercept 
$\Delta_{\mathbb{I P}}$ form the total structure fucntion measurements in the kinematical range of HERA. However, the same Fig. 2 shows that one can zoom at the perturbative cross section at $r \sim 0.15 \mathrm{f}$ measuring excitation of charm at $Q^{2} \lesssim 10^{2}$, so that in this case the effective intercept $\Delta_{e f f}\left(x, Q^{2}\right)=-\partial \log F_{2}\left(x, Q^{2}\right) / \partial \log x \approx \Delta_{\mathbb{P}}=0.4$. At larger $Q^{2}, \Delta_{e f f}\left(x, Q^{2}\right)$ overestimates $\Delta_{\mathbf{I P}}$ because of the double-leading-logarithm efects [7]. Our predictions for the charm structure function are shown in Fig. 3c (The threshold effects in excitation of heavy flavours at small $Q^{2}$ [20] can approximately be taken into account by the rescaling $\left.\xi \Longrightarrow \xi+\log \left(1+\frac{m_{c}^{2}}{Q^{2}}\right)\right)$. Once the pomeron intercept and the perturbative dipole cross section are determined, one can separate the nonperturbative contribution from the high-accuracy data on $F_{2}\left(x, Q^{2}\right)$ at relatively low $Q^{2} \lesssim 20 \mathrm{GeV}^{2}$. To this end, the important point is that at $Q^{2} \gtrsim(10-20) \mathrm{GeV}^{2}$ the BFKL evolution and the conventional GLDAP (Gribov-LipatovDokshitzer-Altarelli-Parisi) [17] evolution complemented by the proper boundary condition, yield essentially identical predictions for the $F_{2}^{(p t)}\left(x, Q^{2}\right)$ in the HERA region (the deep reasons for this similarity are discussed in detail in [7]).

Above we only presented the results for the bare pomeron. At large $\frac{1}{x}$ the unitarization effects become important. They were discussed to great detail in [4,21]. The unitarity bound can best be formulated in terms of the profile function (the partial wave amplitude) $\Gamma(\xi, r, b)$ for the dipole cross section

$$
\Gamma(\xi, r, b) \approx \frac{\sigma(\xi, r)}{4 \pi B_{0}(\xi, r)} \exp \left[-\frac{b^{2}}{2 B_{0}(\xi, r)}\right],
$$

where $\vec{b}$ is the impact parameter. The diffraction slope $B_{0}(\xi, r)$ is a slow function of $\xi$ and $r$ compared to the rapidly changing $\sigma(\xi, r)$. Evidently, the $s$-channel unitarity constraint $\Gamma(\xi, r, b)<1$ will first be violated at large $r$. To a good approximation, the unitarization effects reduce to a (very weakly $Q^{2}$ dependent) renormalization factor $(1-\delta \xi) \sim \exp (-\delta \xi)$ with $\delta \sim 0.03-0.05[4,21]$, which is negligibly small compared to $\Delta_{\mathbf{I P}}=0.4$. Furthermore, in [4] we have shown that the unitarized structure function still satisfies the linear GLDAP evolution. Consequently, for the purposes of the present crude phenomenology, the unitarization effects can be reabsorbed into the (as yet unknown) intercept of the pomeron.

The ratio $R=\sigma_{L} / \sigma_{T}$ was first evaluated in the framework of the dipole cross section technique in [1]. In [21] we have shown that the unitarization effects have little impact on 
$\sigma_{L} / \sigma_{T}$. Our predictions for $R$ for the sea component of the structure function are shown in Fig. 6. We find a sort of crossover at $x \sim(1-2) \cdot 10^{-3}$. At small $x, R$ rises a little with $Q^{2}$, and is essentially flat at $Q^{2} \gtrsim 20 \mathrm{GeV}^{2}$. The knoweledge of $R$ at small $Q^{2}$ is important for certain applications like an accurate estimate of the radiative corrections. In Fig. $6 \mathrm{~b}$ we separately show our predictions for $R$ at $Q^{2} \leq 0.75 \mathrm{GeV}^{2}$. Notice that $\left|\Psi_{L}\right|^{2} \propto Q^{2}$, and at very small $Q^{2}$ we have $R \propto Q^{2}$ just by virtue of gauge invariance.

The relationship between our dipole-cross section representation for deep inelastic scattering and the more familar parton model (recall that the latter comes along with the Weizsäcker-Williams reinterpretation of the photoabsorption cross section [17]) is established by $[3,4,21,22]$

$$
\sigma(\xi, r) \approx \frac{4 \pi}{3} \alpha_{S}(r) \int \frac{d^{2} \vec{k}}{k^{2}} \cdot \frac{1-\exp (i \vec{k} \vec{r})}{k^{2}} \cdot \frac{d G\left(\xi, k^{2}\right)}{d \log k^{2}} \approx \frac{\pi^{2}}{3} r^{2} \alpha_{S}(r) G_{p t}\left(\xi, Q^{2} \sim \frac{1}{r^{2}}\right),
$$

where $G\left(\xi, Q^{2}\right)=x g\left(x, Q^{2}\right)$ is the gluon structure function at $x=x_{0} \exp (-\xi)$. Then, assuming $N_{f}=4$ active flavours, the differentiation of Eq. (10) gives (see also [23])

$$
\frac{\partial F_{2}\left(x, Q^{2}\right)}{\partial \log Q^{2}}=\left(\sum_{u, d, s, c} e_{f}^{2}\right) \frac{\alpha_{S}\left(Q^{2}\right)}{3 \pi} G\left(2 x, Q^{2}\right),
$$

where $e_{i}$ is the quark charge in units of the electron charge. Strictly speaking, Eq. (12) was derived for the perturbative dipole cross section, but one may use Eq. (12) in conjunction with Eq. (13) as a definition of the small- $x$ gluon stucture function beyond the perturbation theory. If we apply the procedure (13) to our BFKL structure functions, then we find $G\left(x, Q^{2}\right)$ shown in Fig. 7 in comparison with the recent H1 [24] and NMC [18] determinations of $G\left(x, Q^{2}\right)$. We have a good overall agreement with the experiment.

\section{Discussion of the results and conclusions:}

We presented the BFKL phenomenology of deep inelastic scattering at large $\frac{1}{x}$ in the dipolecross section representation. The nover features of our approach, which make it different from the related work by other authors [25], are as follows: Firstly, its irrefutable advantage is an exact diagonalization of the $S$-matrix in the dipole-cross section representation. Secondly, the general idea, formulated and exploited in our early works [1-3,20-22], was to use the universality property of the dipole cross section to fix the normalization and the shape 
of $\sigma(\xi, r)$ at moderately large energies. Thirdly, the the nonperturbative dipole cross section which emerges as an integral part of the formalism, is well constrained by the hadronic cross sections [1], the small- $Q^{2}$ structure functions [1,3], the diffraction dissociation of photons [2], the nuclear shadowing [3] and the leptoproduction of vector mesons [15,16]. Fourthly, the discussion in [25] centered on matching the GLDAP solutions with the fixed- $\alpha_{S}$ solutions of the scaling BFKL equation [8]. Solutions of our generalized BFKL equation, which consistently treats the running coupling $\alpha_{S}(r)$, are dramatically different from the fixed- $\alpha_{S}$ solutions. (for the more detailed discussion see [7]). Finally, our infrared regularization is consistent with gauge invariance [4-6]

In our earlier work [1-3,20-22] we tried to describe the whole dipole cross section $\sigma(\xi, r)$ purely perturbatively. As far as the absolute value of $\sigma(\xi=0, r)$ is concerned, that can be achieved at the expense of small $\mu_{G}$. Such a perturbative glue always starts with $g\left(x, Q^{2}\right) \propto$ $\frac{1}{x}$ and always generates the sea $\bar{q}\left(x, Q^{2}\right) \propto \frac{1}{x}$, providing a nice continuity from the real photoproduction to deep inelastic scattering. The trouble with small $\mu_{G}$ is a too rapid a buildup of the perturbative glue, which can be tamed, and a good quantitative description of the ZEUS and H1 structure functions can be obtained, at the expense of starting the GLDAP evolution of glue at $Q_{0}^{2} \approx 1 \mathrm{GeV}^{2} \gg 4 \mu_{G}^{2}$ [21] (the unitraization effects were also found important to bring the predictons [21] closer to the ZEUS and H1 data). There is much correspondance between this large value of $Q_{0}^{2}$ of Ref. 21 and the small gluon correlation radius $R_{c}$ used in the present paper. The present analysis shows that in order to understand the HERA data, one really needs a large nonperturbative dipole cross besides the perturbative BFKL cross section.

We conclude listing the uncertanties and challenges: The pomeron intercept and the pomeron cross section are evidently sensitive to the specific choice of the gluon correlation radius $R_{c}$ and of the freezing coupling, and also can change if more sophisticated infrared regularization is introduced. Another uncertainty is the choice of $x_{0}$. Hovever, none of the above will drastically modify the salient features of the above presented dipole-cross section phenomenology . The further progress in the theory requires the experimental determination of the pomeron intercept $\Delta_{\mathbb{I}}$, which the HERA experiments on excitation of charm can do, 
and better understanding of the nonperturbative cross section, which also can be probed in the HERA experiments. From the theoretical side, more work on the unitarization effects is in order.

Acknowledgements: B.G.Z. is grateful to J.Speth for the hospitality at IKP, KFA Jülich, where this work was initiated. 


\section{References}

[1] N.N. Nikolaev and B.G. Zakharov, Z. Phys. C49 (1991) 607.

[2] N.N. Nikolaev and B.G. Zakharov, Z. Phys. C53 (1992) 331.

[3] V.Barone, M.Genovese, N.N.Nikolaev, E.Predazzi and B.G.Zakharov, Z. Phys. C58 (1993) 541

[4] N.N.Nikolaev and B.G.Zakharov, The triple-pomeron regime and the structure function of the pomeron in the diffractive deep inelastic scattering at very small $x$, Landau Inst. preprint Landau-16/93 and Jülich preprint KFA-IKP(Th)-1993-17, June 1993, submitted to Z. Phys. C.

[5] N.N.Nikolaev, B.G.Zakharov and V.R.Zoller, The $s$-channel approach to Lipatovs pomeron and hadronic cross sections, Jülich preprint KFA-IKP(TH)-1993-34, December 1993; JETP Letters, to be published.

[6] N.N.Nikolaev, B.G.Zakharov and V.R.Zoller, The spectrum and solutions of the generalized BFKL equation for total cross section, Jülich preprint KFA-IKP(TH)-19941, January 1994; submitted to Phys. Lett. B.

[7] N.N.Nikolaev and B.G.Zakharov, BFKL evolution and universal structure function at very small $x$, Jülich preprint KFA-IKP(TH)-1994-1, January 1994; submitted to Phys. Lett. B.

[8] E.A.Kuraev, L.N.Lipatov and V.S.Fadin, Sov.Phys. JETP 44 (1976) 443; 45 (1977) 199; Ya.Ya.Balitskii and L.N.Lipatov, Sov. J. Nucl. Phys. 28 (1978) 822; L.N.Lipatov, Sov. Phys. JETP 63 (1986) 904; L.N.Lipatov. Pomeron in Quantum Chromodynamics. In: Perturbative Quantum Chromodynamics, editor A.H.Mueller, World Scientific, 1989

[9] M.Derrick et al., ZEUS Collaboration, Phys. Lett. B (1993).

[10] I.Abt et al., H1 Collaboration, Nucl. Phys. B407 (1993) 515. 
[11] E.Shuryak, Rev. Mod. Phys. 65 (1993) 1, and references therein.

[12] For the similar phenomenology of the $(\bar{p}) p-p$ total cross section see B.Z.Kopeliovich, N.N.Nikolaev and I.K.Potashnikova, Phys. Rev D39 (1989) 769.

[13] G.Fang, E665 preliminary results reported at PANIC, Perugia, June 1993.

[14] C.Mariotti, Proceedings of the Joint International Lepton-Photon Symposium and Europhysics Conference on High-Energy Physics, 25 July - 1 August 1991, Geneva. Eds. S.Hegarty, K.Potter and E.Quercigh, World Scientific, v.1, p.154.

[15] B.Z.Kopeliovich and B.G.Zakharov, Phys. Rev. D44 (1991) 3466; O.Benhar, B.Z.Kopeliovich, C.Mariotti, N.N.Nikolaev and B.G.Zakharov,Phys. Rev.Lett. 69 (1992) 1156

[16] B.Z.Kopeliovich, J.Nemchik, N.N.Nikolaev and B.G.Zakharov, Phys. Lett. B309 (1993) 179; Decisive test of colour transparency in exclusive electroproduction of vector mesons, Jülich preprint KFA-IKP(TH)-1993-29, Phys. Lett. B, to be published.

[17] V.N. Gribov and L.N. Lipatov, Sov. J. Nucl. Phys. 15 (1972) 438; L.N. Lipatov, Sov. J. Nucl. Phys. 20 (1974) 181; Yu.L. Dokshitser, Sov. Phys. JETP 46 (1977) 641; G. Altarelli and G. Parisi, Nucl. Phys. B126 (1977) 298.

[18] NMC collaboration: M.Arneodo et al., Phys. Lett. B309 (1993) 222.

[19] For the Regge-theory motivated fits to structure functions see A.Donnachie and P.V.Landshoff, M/C-th 93/11, DAMPT 93-23.

[20] V.Barone, M.Genovese, N.N.Nikolaev, E.Predazzi and B.G.Zakharov, Phys.Lett. B264 (1991) 279.

[21] V. Barone, M. Genovese, N.N. Nikolaev, E. Predazzi and B.G. Zakharov, Torino preprint DFTT 28/93, June 1993, submitted to Phys.Lett. B.

[22] V.Barone, M.Genovese, N.N.Nikolaev, E.Predazzi and B.G.Zakharov, Int.J.Mod.Phys A, 8 (1993) 2779. 
[23] R.G.Roberts, The structure of the proton. (Cambridge Univ. Press, 1990), section 6.4; K.Prytz, Phys. Lett. B311 (1993) 286.

[24] I.Abt et al., H1 Collaboration, DESY 93-146.

[25] A.J.Askew, J.Kwiecinski, A.D.Martin and P.J.Sutton, Phys. Rev. D47 (1993) 145; Durham preprint DTP/93/28 and references therein. 


\section{Figure captions:}

Fig.1 - The nonperturbative and perturbative components of the dipole cross section.

Fig.2 - The kernel $\Phi_{T}\left(Q^{2}, r^{2}\right)$ for (the top box) the light $(u, d)$ and (the bottom box) the charmed quarks.

Fig.3 - The predicted BFKL structure functions at small $x$ : (a) the total structure function, (b) the perturbative part of the structure function, (c) the charm structure function. The curves correspond to (from bottom to top) $Q^{2}=0.75,1.25,1.75,2.5,3.5,4.5,8.5,-$ 15, 30,60, 120, 240, $480 \mathrm{GeV}^{2}$, respectively.

Fig.4 - Comparison of the predicted BFKL structure functions with the data from the ZEUS [10], H1 [11] and NMC [18] experiments.

Fig.5 - Predictions for $R=\sigma_{L} / \sigma_{T}$. The lower box shows the low- $Q^{2}$ behavior of $R$.

Fig.6 - Comparison of the gluon structure function evaluated from the predicted BFKL structure functions using Eq. (13) with the H1 [24] and NMC [18] determinations. 
This figure "fig1-1.png" is available in "png" format from: http://arxiv.org/ps/hep-ph/9402209v1 
This figure "fig2-1.png" is available in "png" format from: http://arxiv.org/ps/hep-ph/9402209v1 
This figure "fig1-2.png" is available in "png" format from: http://arxiv.org/ps/hep-ph/9402209v1 
This figure "fig2-2.png" is available in "png" format from: http://arxiv.org/ps/hep-ph/9402209v1 
This figure "fig1-3.png" is available in "png" format from: http://arxiv.org/ps/hep-ph/9402209v1 
This figure "fig2-3.png" is available in "png" format from: http://arxiv.org/ps/hep-ph/9402209v1 